CrossMark $\leftarrow$ click for updates

Cite this: Phys. Chem. Chem. Phys., 2015, 17, 28774

Received 10th July 2015 , Accepted 20th September 2015

DOI: $10.1039 /$ c5cp04028k

www.rsc.org/pccp

\title{
Spectroscopic ellipsometry meets AFM nanolithography: about hydration of bio-inert oligo(ethylene glycol)-terminated self assembled monolayers on gold
}

\author{
Ilaria Solano, ${ }^{a}$ Pietro Parisse, ${ }^{\mathrm{b}}$ Federico Gramazio, ${ }^{a}$ Ornella Cavalleri, ${ }^{a}$ \\ Gianangelo Bracco, ${ }^{a}$ Matteo Castronovo, ${ }^{\mathrm{C}}$ Loredana Casalis ${ }^{\mathrm{b}}$ and \\ Maurizio Canepa*a
}

\begin{abstract}
For the first time, to our knowledge, spectroscopic ellipsometry (SE) has been combined with state-ofthe-art AFM differential height measurements conducted after shaving nano-lithography of ultrathin, soft-matter films for thickness determination. We investigated self-assembled monolayers of $\mathrm{SH}-$ $\left(\mathrm{CH}_{2}\right) 11-\mathrm{EG}_{n}-\mathrm{OH}$ molecules on gold, where $\mathrm{EG}$ is ethylene glycol units and $n=3$ and 6 , a prototypical non-fouling system. We performed SE measurements $(245-1200 \mathrm{~nm})$ focusing on the changes induced by the formation of the film (difference spectra). SE measurements, analysed by simple models, confirm the formation of the S-Au interface, transparency of the SAMs and provide a sharp picture of the ability of the EG functionality to protect the surface from unspecific adsorption of proteins. A quantitative assessment of the film thickness by SE was carried out ex situ, thanks to the optical contrast between the film and the ambient, and by AFM in liquid. The cross-check between SE and AFM height measurements combined with the comparison between in-liquid and ex situ SE measurements allowed obtaining non-perturbative information about the vertical density profile of the SAM. The in-liquid SE measurements indicate a refractive index matching between the aqueous medium and the outer part of the SAM, consistent with a disordered configuration of OEG and/or the penetration of water amid the OEG strands. A critical discussion provides a detailed insight into the subtle issues and pitfalls related to the thickness determination of soft-matter films to the monolayer limit.
\end{abstract}

\section{Introduction}

The progress of bioanalytical technologies based on the integration of sensors with micro- and nano-electro-mechanical devices requires the development of label-free and non-invasive tools for the accurate characterization of sensing surface elements. In this context, organic or bio-functionalization (for example, by self assembled monolayers, SAMs) of solid supports plays a pivotal role. ${ }^{1,2}$ In general, biomolecular adsorption on a surface can be determined by measuring the inherent mass transfer, or, alternatively, the inherent increase of thickness.

Light underlies the most gentle analytical methods of organic and biologic ultrathin films and SAMs. Optical measurements can be performed in transparent liquid media such as the aqueous

\footnotetext{
${ }^{a}$ Dipartimento di Fisica, Università di Genova and CNISM, via Dodecaneso 33, Genova, Italy.E-mail: canepa@fisica.unige.it

${ }^{b}$ Elettra Sincrotrone Trieste S.C.p.A., s.s. $14 \mathrm{~km} \mathrm{163,} 5$ in Area Science Park, Basovizza, Trieste, Italy

${ }^{c}$ Department of Medical and Biological Sciences - University of Udine, Udine, Italy
}

environment that is associated with biological samples, and in a time-resolved fashion. Ellipsometry, as well as SPR measurements, are commonly exploited for monitoring adsorption/desorption processes, ${ }^{3,4}$ eventually in a combined fashion as in total internal reflection ellipsometry. ${ }^{5,6}$ Ellipsometry, in particular, is popularly used to quickly and effectively estimate the SAM thickness since pioneering experiments in the field were established. ${ }^{7-9}$ In contrast to SPR, ellipsometry is in principle applicable to a number of solid supports with adequate reflectivity.

Specifically, optical techniques provide indirect measurements of the thickness and other structural parameters of films. Within the frame of Fresnel optics, for ultra-thin films (film thickness $d \ll \lambda$ ), specifically in the range of transparency, the thickness is increasingly correlated to the refractive index, ${ }^{10,11}$ which is known only approximately in general. It is therefore convenient to combine ellipsometry with other methods. ${ }^{12-14}$

Atomic force microscopy (AFM) methods represent an independent integration to many kinds of optical spectroscopy measurements. ${ }^{15-17}$ In shaving nano-lithography, ${ }^{18}$ for instance, exploiting the removal of molecules by the high shear force 
exerted by the tip during an areal scan, one can perform differential height measurements between fully-covered and fully-depleted, adjacent areas. Shaving is therefore particularly appealing as it allows examining compact SAMs that are prepared under conditions basically similar to those adopted for ellipsometry measurements; furthermore, such kinds of measurements offer an additional complementarity as to the lateral resolution, at the micro-scale (AFM) vs. macro-scale (SE).

In this work, to our knowledge, for the first time, state-ofthe-art spectroscopic ellipsometry (SE) and AFM nanolithography are combined to obtain detailed information on the thickness and optical properties of antifouling SAMs that are typically utilized to reduce the adsorption background of surfacebased biosensors. ${ }^{2,19}$

We focus on oligo(ethylene glycol)-terminated alkanethiol (for brevity, T-OEG) SAMs on gold surfaces, whose intriguing non-fouling properties were early emphasized in seminal studies. ${ }^{7,1}$ While T-OEG molecules form homogeneous and uniform SAMs over large areas, they are locally poorly organized compared to the most stable alkane SAMs, mostly due to the interactions between flexible OEG strands. ${ }^{20-26}$

Such an intrinsic and local disorder is believed to play a role in determining the interaction between the OEG moieties and water molecules, effective in preventing the underlying methylene groups from wetting by water. ${ }^{7}$ There is also an accumulating consensus on the manifold role of the water-OEG interaction, and in particular of the penetration of water into the SAM and the related hydration of the OEG part in establishing the bio-inertness of these SAMs, ${ }^{27-33}$ which also favours their stability in room ambient.

Furthermore, the relatively poor organization of T-OEG SAMs makes simple isotropic models rather adequate to their optical modelling.

On the practical side of SE measurements, we adopted an approach which is based on the analysis of small changes in the ellipsometric response upon formation of the film. Our somewhat unconventional approach to the analysis of difference spectra $^{12,34,35}$ allows disentanglement of spectral features related to the SAM and the SAM-substrate interface; ${ }^{36,37}$ the method also provides fingerprints of molecular UV-Vis absorption ${ }^{38}$ thus extending the traditional spectroscopic ellipsometry to provide a sensitive solution to surface UV-Vis spectroscopy. ${ }^{39-42}$

Our approach helps defining the sources of systematic uncertainty associated with both SE and differential AFM measurements, an aspect which is not adequately addressed in routine applications of the individual methods. The combination of difference ellipsometry spectra and differential AFM measurements considerably strengthens the reliability of the thickness determination proposed in this work. The cross-check between SE and AFM, and the comparison between wet and dry samples allow obtaining non-perturbative information regarding the vertical density profile of the SAM and the penetration of water amid the OEG chains. Last but not least, improving the accuracy in the determination of the thickness opens the door to obtaining more reliable estimates of the refractive index of the films, a piece of information that is elusive for such ultrathin layers.

\section{Materials and methods}

\subsection{Materials}

Gold substrates and SAM preparation. AFM measurements were performed on the so-called Ulman ultra-flat gold substrates, ${ }^{43}$ prepared following a modified version of the stripping procedure described in detail in previous papers. ${ }^{44}$ The stripped substrates presented a typical surface roughness of $0.2 \mathrm{~nm}$ as measured by AFM on $5 \times 5 \mu^{2}$ areas. The samples were then soaked in a $300 \mu \mathrm{M}$ solution of T-OEG in ethanol for about 24 hours, to make it ready for the nanoshaving procedure.

For optical measurements we used commercial (Arrandee) gold films deposited on glass with a Cr primer. ${ }^{34}$ All the substrates were treated by chemical etching (60\% MilliQ water, $30 \%$ ammonia solution and $30 \%$ hydrogen peroxide at $100{ }^{\circ} \mathrm{C}$ for $10 \mathrm{~min}$ ), rinsed in MilliQ water and fluxed with $\mathrm{N}_{2}$ and characterized by ex situ SE. The optical properties of substrates were consistent with previous determinations. ${ }^{34,45}$ A subset of substrates was also characterized by SE in TE buffer. After incubation in the T-OEG solution in ethanol $\left(4{ }^{\circ} \mathrm{C}, 18 \mathrm{~h}\right)$ the samples were extracted and rinsed with ethanol, dried under $\mathrm{N}_{2}$ flow and immediately characterized by ex situ SE. For subsets of samples, ethanol was substituted by TE buffer or MilliQ water for in situ SE characterization and test of the resistance properties. A representative set of T-OEG SAMs were exposed to a $1 \mu \mathrm{M}$ YCC-Milli-Q solution for $2 \mathrm{~h}$ at $4{ }^{\circ} \mathrm{C}$. The molecular solution was then gradually substituted with pure Milli-Q water and the sample was characterized by in situ and ex situ SE, after being dried under $\mathrm{N}_{2}$ flow.

Reagents. (1-Mercaptoundec-11-yl)tri(ethylene glycol) (SH$\left(\mathrm{CH}_{2}\right)$ 11-EG 3 -OH, T-OEG3) and (1-mercaptoundec-11-yl)hexa(ethylene glycol) $\left(\mathrm{SH}-\left(\mathrm{CH}_{2}\right) 11-\mathrm{EG}_{6}-\mathrm{OH}, \mathrm{T}-\mathrm{OEG} 6\right)$ were purchased from Prochimia. Note that the two thiols have the same alkyl C11 chain and differ in the length of the ethylene-glycol (EG, $\mathrm{OCH}_{2} \mathrm{CH}_{2}$ ) residual.

Cytochrome $C(\mathrm{YCC})$ (104 residues, $M_{\mathrm{W}} 12.4 \mathrm{kDa}$, size about $4 \mathrm{~nm}$ ) was used as received from Sigma Aldrich. TE buffer was prepared using sodium chloride ( $\mathrm{NaCl})$, Tris(hydroxymethyl)aminomethane and ethylenediaminetetraacetic acid (EDTA) from Sigma Aldrich diluted in Milli-Q water (Millipore, resistivity $18.2 \mathrm{M} \Omega \mathrm{cm})$.

\subsection{Methods}

Spectroscopic ellipsometry. Principles of SE are described at length in books. ${ }^{10,11,46}$ Recent reviews were oriented to nanoscience applications. ${ }^{47,48}$ The so-called standard ellipsometry investigates the coefficient $\rho=\tilde{r}_{\mathrm{p}} / \tilde{r}_{\mathrm{s}}=\tan \Psi \exp (i \Delta)$, where $\tilde{r}_{\mathrm{p}}$ and $\tilde{r}_{\mathrm{s}}$ are the complex Fresnel reflection coefficients for p- and s-polarization, respectively. Data are analysed by comparison with simulations based on an optical and morphological model of the layered system. The value of convenient adjustable parameters is derived by fitting experimental spectra and simulations, exploiting fit quality estimators such as the mean squared error, MSE, function. ${ }^{49,50}$

The measurements were obtained using a rotating compensator spectroscopic ellipsometer (M-2000, J.A. Woollam Co. Inc.) equipped 
with a $75 \mathrm{~W}$ Xe lamp. Ex situ spectra of dry samples have been collected at $65-70^{\circ}$ of incidence and are reported in the 245 $1200 \mathrm{~nm}$ interval where difference spectra showed a satisfactory signal-to-noise ratio. In-liquid measurements were performed in a home-made Teflon cell described in ref. 51 (angle of incidence $\left.65^{\circ}\right)$ and data are reported in the range of high transparency of the medium. The spot size on the sample was of the order of a few $\mathrm{mm}^{2}$. We focus on the difference spectra method ${ }^{10,52,53}$ thoroughly described in recent articles on ultrathin layers. ${ }^{34,37-39}$ $\delta \Psi$ and $\delta \Delta$ will denote the difference between the data obtained for SAM-covered and pristine substrates $\left(\delta \Psi=\Psi_{\text {film }+\mathrm{Au}}-\Psi_{\mathrm{Au}}\right.$ and $\delta \Delta=$ $\left.\Delta_{\text {film }+\mathrm{Au}}-\Delta_{\mathrm{Au}}\right)$. Difference spectra are presented after averaging over not less than five samples and typically over four different zones of each sample.

Atomic force microscopy: nanoshaving and height measurements. AFM experiments were carried out using XE100 (Park Instruments) and MFP-3D (Asylum Research) instruments in custom-designed liquid cells. For nanoshaving, sketched in the upper part of Fig. 1, we operated in contact mode in pure ethanol with commercial AFM tips (NSC19 Mikromasch, $\left.k=0.6 \mathrm{~N} \mathrm{~m}^{-1}\right)$. During the shaving process a high load (100 nN) was applied to a small $\left(0.5 \times 0.5-1 \times 1 \mu^{2}\right)$ scanning area. The lateral movement of the tip during the scanning at high force allows the local displacement of the molecules linked to the surface (Fig. 1A and B). The molecules will then diffuse into the solution, leaving the gold surface exposed in the shaved area (Fig. 1C).

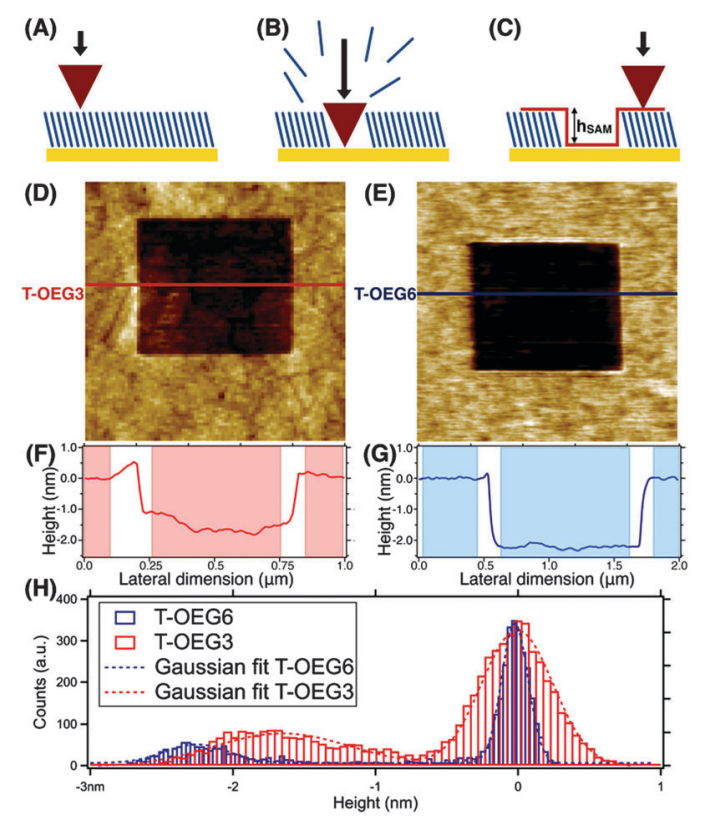

Fig. 1 (A-C) The nanoshaving process: applying a high load the AFM tip locally removes the SAM molecules. The process produces depleted areas ready for height measurements. ( $D$ and $E$ ) AFM micrographs and ( $F$ and $G$ ) representative height profiles across shaved regions in T-OEG SAMs. The edges of the shaved areas have been excluded from the process of height evaluation as suggested by the shading in panels $F$ and $G$. $(H)$ Height histograms for nanoshaved areas in T-OEG3 and T-OEG6, with the corresponding Gaussian fits (dotted lines). Histograms have been aligned setting arbitrarily the zero at the SAM surface.
The depth of the shaved areas $\left(h_{\mathrm{SAM}}\right)$ provided an accurate estimate of the film thickness in a liquid. $h_{\text {SAM }}$ was measured from both line profiles and height histogram analyses as the difference between the top of the molecular layer and the bottom of the shaved area (Fig. 1C). Regarding line profiles, we took the average value of the height of the SAM surface and subtracted the average value of the height of the gold surface, being careful to avoid the edges of the shaved area as suggested by shading in Fig. 1F and G. The larger surface roughness of the nanoshaved areas with respect to the surrounding SAM carpet is due to the nanoshaving process that can slightly damage the surface and/or leave the residual molecules on the depleted surfaces. The height histograms were calculated for every image independently, by analysing the $256 \times 256$ or $512 \times 512$ $z$-coordinate values in the topographic maps. Height histograms have been aligned setting arbitrarily the zero at the SAM surface. The two methods provided comparable results. We will focus in Section 3.1 on the height histogram analysis.

Topographic images were acquired in soft contact mode in saline buffer (10 mM Tris-HCl, 1 mM EDTA, $1 \mathrm{M} \mathrm{NaCl}, \mathrm{pH}=7.2$ ) using softer commercial cantilevers (CSC38 Mikromasch, $\left.k=0.03 \mathrm{~N} \mathrm{~m}^{-1}\right)$ at the minimum force detectable $(0.1 \mathrm{nN})$. This minimum force allows a soft contact on the surface and maximally reduces the effect of the applied load of the tip on the molecules. Piezoscanners and cantilevers were carefully calibrated before the nanoshaving sessions, and the non-linear effects are corrected through a feedback loop system (internal to the AFM setups) in both the $X-Y$ plane and the $Z$-direction. Images and data were analyzed using XEI (Park Instruments), Igor Pro (Wavemetrics Inc.) and Gwyddion (Gwyddion.net) softwares. The measurements on dried samples (images not shown) were affected by stray adsorption in the depleted regions, even after careful washing in pure ethanol. However, while the depleted areas were contaminated, the SAM surfaces preserved the roughness and conformation measured in a liquid.

\section{Results and analysis}

\subsection{Atomic force microscopy}

Typical AFM images and the corresponding height profiles of shaved areas (in liquid) in T-OEG3 and T-OEG6 SAMs are reported in Fig. 1(D-F) and (E-G), respectively. In panel $\mathrm{H}$, reporting the height histograms relative to the specific images of panels D (T-OEG3) and E (T-OEG6), we can observe two peaks, the one (centered at $0 \mathrm{~nm}$ ) corresponding to the surface of the SAM (brighter areas in panels D and E), and the broader one corresponding to the shaved regions (darker areas). We performed a fit of the height distribution with two Gaussian curves, whose inter-distance, $h_{\mathrm{SAM}}$, can be considered the AFM thickness of the layer. The errors on $h_{\mathrm{SAM}}$ have been evaluated taking into account the sum of standard deviation values derived from the fit relative to the SAM surface and the shaved area. In the specific case reported the values of $h_{\mathrm{SAM}}$ are $1.6 \pm$ $0.6 \mathrm{~nm}$ for T-OEG3 and $2.2 \pm 0.4$ for T-OEG6. From the statistical analysis of the $h_{\mathrm{SAM}}$ values of different shaved areas 
in several samples, we obtained an experimental thickness of $1.6 \pm 0.4 \mathrm{~nm}$ and $2.4 \pm 0.3 \mathrm{~nm}$ for the T-OEG3 and T-OEG6 SAMs, respectively.

\subsection{Spectroscopic ellipsometry}

The ex situ SE data on T-OEG films are shown in Fig. 2. The reported simulations were calculated according to the simplest model (TF $\mid \mathrm{S})$ in which a transparent film (TF) is sharply stacked onto the substrate (S). The film was modelled through a simplified Cauchy dispersion formula: ${ }^{10,11,46}$

$$
n=n_{\mathrm{TF}}+\frac{B\left[\mu \mathrm{m}^{2}\right]}{\lambda^{2}} ; \quad \kappa=0 .
$$

Simulations are presented for an indicative range of $n_{\mathrm{TF}}$ as a function of the film thickness $d_{\mathrm{TF}}$. The coefficient $B$ was set to 0.01 . They reproduce the shape of experimental spectra with the relevant exception of the negative $\delta \Psi$ values in the high reflectivity region of gold $\left(\approx-0.1^{\circ}\right.$ at $65^{\circ}$ and $\lambda=750 \mathrm{~nm}$ for both SAMs) comparable to those found for other thiolate SAMs. ${ }^{34,36}$ No intrinsic molecular optical absorption (observed as
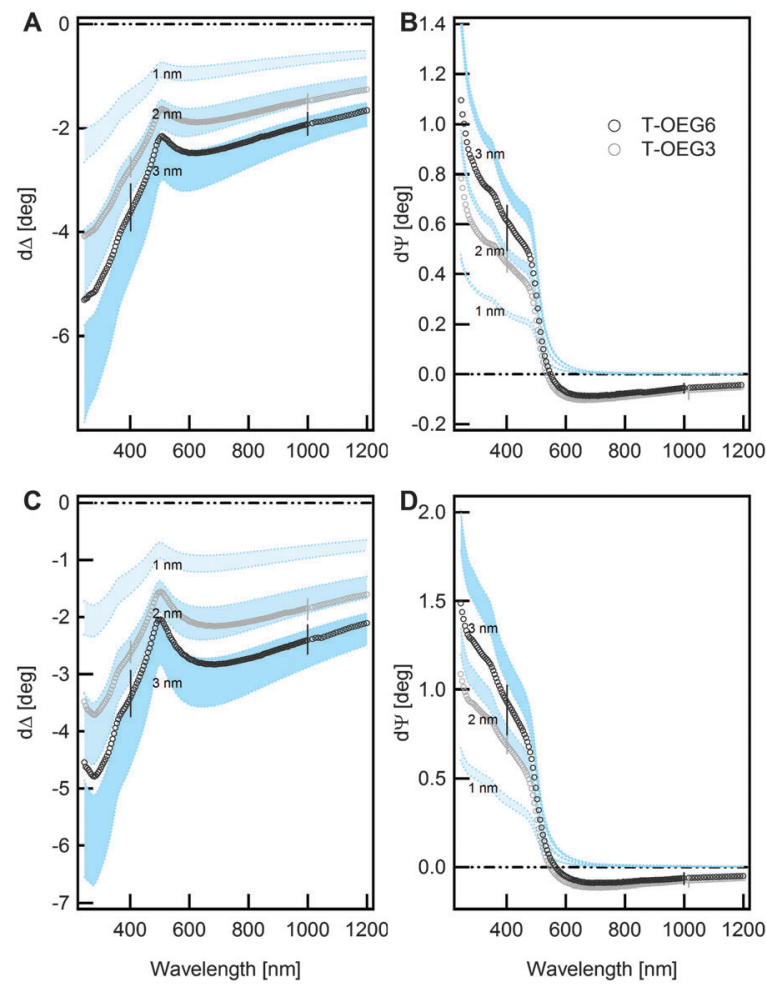

Fig. 2 Open circles (grey tones): ex situ spectroscopic ellipsometry data (difference spectra: $\delta \Psi=\Psi_{\text {film+Au }}-\Psi_{\mathrm{Au}} \delta \Delta=\Delta_{\text {film+Au }}-\Delta_{\mathrm{Au}}$ ) obtained for the T-OEG SAMs on Au at ( $A$ and $B$ ) $65^{\circ}$ and (C and D) $70^{\circ}$ angles of incidence. Thin vertical bars show representative uncertainty in different spectral regions. Shaded areas illustrate simulations based on the TF|S model, for three values of the SAM thickness $\left(d_{\mathrm{TF}}=1.0,2.0\right.$ and $\left.3 \mathrm{~nm}\right)$ and provide a graphical representation of the index/thickness correlation. For $\delta \Delta$ patterns (left) the top and bottom borders of shading correspond to $n_{\mathrm{TF}}=1.35$ and 1.55 , respectively; for $\delta \Psi$ spectra (right panels) the order is reversed. For a given $n_{\mathrm{TF}}$, simulated $\delta \Delta$ curves are proportional to $d_{\mathrm{TF}}$. The $\mathrm{TF} \mid \mathrm{S}$ model reproduces the shape of both $\delta \Delta$ and $\delta \Psi$ spectra with the exception of the negative $\delta \Psi$ values above $550 \mathrm{~nm}$ sharp dips in difference spectra of other SAMs on $\mathrm{Au}^{38,40}$ ) was discernible.

For ultrathin transparent films of given $n_{\mathrm{TF}}$ the proportionality between $\delta \Delta$ and $d_{\mathrm{TF}}$ is used in routine checks of thickness, which usually assume some convenient values of the refractive index and ignore the interface effects. ${ }^{7,54}$ The thickness of T-OEG3 and T-OEG6 SAMs that can be extrapolated from panels $A$ and $C$ appears slightly larger than the values obtained from AFM.

Simulated $\delta \Psi$ curves (panels B and D) at low $\lambda$, before the evident step-like transition, are proportional to the thickness as well. The thickness is much less dependent on the value of $n_{\mathrm{TF}}$ and one can easily extrapolate $d_{\mathrm{TF}}$ values in agreement with AFM measurements.

The apparent discrepancy between the $d_{\mathrm{TF}}$ values indicated by the disjunct analysis of $\delta \Delta$ and $\delta \Psi$ data as well as the negative $\delta \Psi$ values witnesses the limitations of the TF|S model. $\delta \Psi$ negative values of thiolate films were indeed attributed, after optical reflectivity studies, ${ }^{55}$ to the film/substrate interface. ${ }^{34}$ This interpretation found support in our recent work on selenide SAMs where effects induced by the formation of the $\mathrm{Se}-\mathrm{Au}$ bond were even stronger than in the S-Au case. ${ }^{37}$

Therefore a more precise evaluation of the film thickness needs the inclusion of interface effects in the optical model. ${ }^{37}$

Within the Fresnel approach, a quick way to account for the interface is the addition of a so-called transition layer between the substrate and the SAMs (TF $|\mathrm{I}| \mathrm{S}$ model). Following a wellknown approach, ${ }^{56}$ the dielectric function of the transition layer is approximated through the Bruggeman effective medium approximation (BEMA):

$$
\sum_{i=1} f_{i} \frac{\varepsilon_{i}-\varepsilon_{e}}{\varepsilon_{i}+2 \varepsilon_{e}}=0
$$

where $\varepsilon_{i}$ and $f_{i}$ are the dielectric functions and volume fractions of the mixing constituents, respectively. The BEMA formula is frequently used to account for the micro-roughness at the vacuum-solid interface. ${ }^{46}$ Analogously, in our context, the BEMA transition layer could be viewed as a kind of interface roughness. The BEMA model depends on two parameters, i.e. the fraction $f_{\text {SAM }}$ and the interface thickness $d_{\mathrm{I}}$. In the simplest approximation $f_{\text {SAM }}$ was set to 0.5 . In the case of gold substrates the introduction of the BEMA transition layer effectively introduces negative $\delta \Psi$ values in the NIR, depending crucially on the value of $d_{\mathrm{I}}{ }^{37}$ The BEMA layer also affects, to a minor extent, the $\delta \Psi$ UV region, and the $\delta \Delta$ spectra as well as it is detailed in Fig. 3, where the effect of $d_{\mathrm{TF}}, d_{\mathrm{I}}$ and $f_{\mathrm{SAM}}$ is analysed separately. In panels $\mathrm{A}$ and $\mathrm{B}$, the increase of $d_{\mathrm{I}}$ values from 0 to $0.4 \mathrm{~nm}$ induces the progressive formation of a negative minimum of $\delta \Psi$ at about $600 \mathrm{~nm}$ and a parallel quasi-rigid downward shift of $\delta \Delta$ related to the increment of the total $d_{\mathrm{TF}}+d_{\mathrm{I}}$ thickness. In panels $\mathrm{C}$ and $\mathrm{D}$, upon increasing $d_{\mathrm{TF}}$ from 1.0 to $3.0 \mathrm{~nm}$, one can note the downward shift of $\delta \Delta$ spectra related to the increase of thickness, and the corresponding increase of $\delta \Psi$ in the UV limit. The increase of $d_{\mathrm{TF}}$ does not significantly affect the negative $\delta \Psi$ data. The effect of $f_{\mathrm{SAM}}$ at fixed $d_{\mathrm{TF}}(2 \mathrm{~nm})$ and $d_{\mathrm{I}}(0.2 \mathrm{~nm})$ is then illustrated in panels $\mathrm{E}$ and $\mathrm{F}$. An increasing $f_{\mathrm{SAM}}$ leads to a broader and broader 

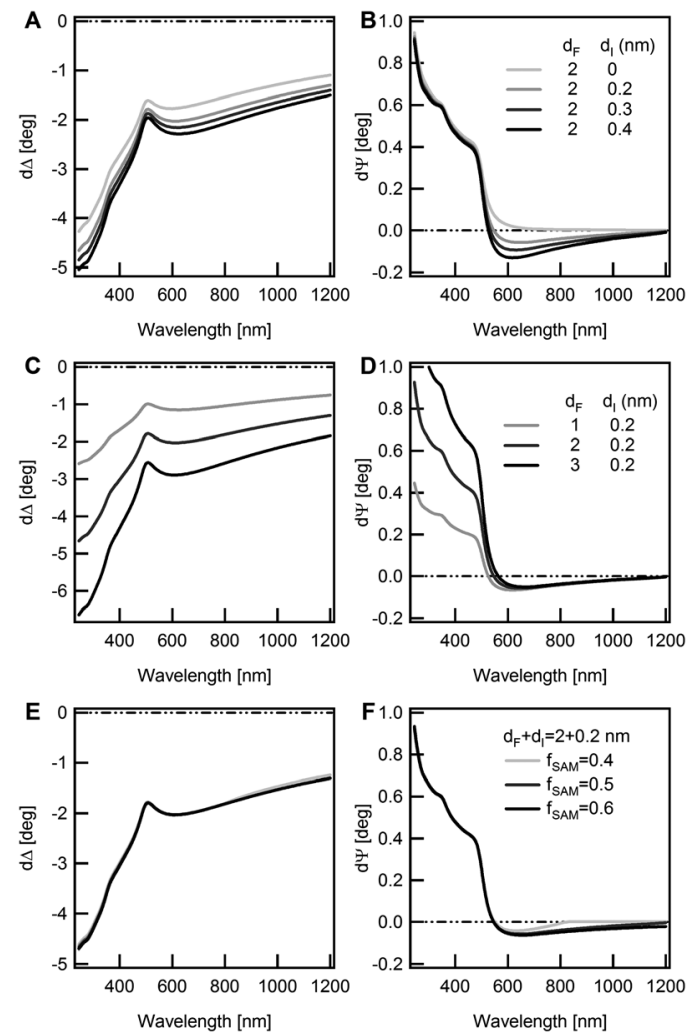

Fig. 3 Simulations of SE data (difference spectra) based on the TF|||S model, in which a transition layer (BEMA approximation) is sandwiched between the substrate and the transparent film. All the simulations were obtained for an indicative value of $n_{\mathrm{TF}}$ (1.4). (A and B) The effect of the variation of the interface thickness $d_{1}$ from 0 to $0.4 \mathrm{~nm}$, at fixed $d_{\mathrm{TF}}(2 \mathrm{~nm})$ and $f_{\text {SAM }}(0.5)$; note the negative $\delta \Psi$ values for $\lambda>550 \mathrm{~nm}$. Instead, the NIR $\delta \Delta$ values depend on the total thickness $d_{\mathrm{TF}}+d_{1}$. (C and D) The effect of the variation of $d_{\mathrm{TF}}$ from 1 to $3 \mathrm{~nm}$, having set $d_{1}$ to $0.2 \mathrm{~nm}$ and $f_{\mathrm{SAM}}$ to 0.5 ; note that NIR $\delta \Psi$ values are not affected by $d_{\text {TF. }}$ ( $E$ and F) The effect of the variation of the interface parameter $f_{\text {SAM }}$, from 0.35 and 0.65 , having set $d_{\mathrm{TF}}$ to $2 \mathrm{~nm}$ and $d_{1}$ to $0.2 \mathrm{~nm}$.

extension of negative $\delta \Psi$ data in the NIR region. The $\delta \Delta$ spectra, and the $\delta \Psi$ values below $550 \mathrm{~nm}$, are substantially untouched.

The main outcomes of Fig. 3 can be summarized as follows: (i) $\delta \Psi$ values below $550 \mathrm{~nm}$ are strongly sensitive to the transparent part of the film (the molecular backbone), (ii) the negative $\delta \Psi$ values above $550 \mathrm{~nm}$ are related only to the interface parameters and (iii) $\delta \Delta$ spectra, throughout the extended spectral range, are sensitive to the total overlayer thickness.

Fitting the $\mathrm{TF}|\mathrm{I}| \mathrm{S}$ model to the experimental data one has to consider the index $v s$. thickness anti-correlation. We initially considered a wide range for the SAM refractive index, $1.25<$ $n_{\mathrm{TF}}<1.7$, with increments of 0.05 . We looked for the MSE minimization by exploiting $B, d_{\mathrm{TF}}, d_{\mathrm{I}}$ and $f_{\mathrm{SAM}}$ as adjustable parameters.

For both T-OEGs, a well-defined minimum of the MSE (around 2-2.5) was found for relatively low values of $n_{\mathrm{TF}}$ (1.35-1.40) with $d_{\mathrm{TF}}=2.6-2.5(1.9-1.8) \mathrm{nm}$ for T-OEG6 (T-OEG3). The MSE decisively increased up to values of the order of 10 for $n_{\mathrm{TF}}=1.7$, where $d_{\mathrm{TF}}$ was about 2(1.4) nm for T-OEG6 (T-OEG3). Only small adjustments of interface parameters were observed; the dispersion parameter $B$ showed a poor variability in the 0.005-0.006 range, values which appear fully comparable to determinations on thicker organic transparent films. ${ }^{57}$ The best reproduction of the negative part of $\delta \Psi$ required values of $d_{\mathrm{I}}$ of about 0.25 for both molecules, with $f_{\mathrm{SAM}}$ of $\approx 60 \%$, comparable to values found on other thiolate films. ${ }^{34}$

In a refining step, we restricted $n_{\mathrm{TF}}$ between 1.35 and 1.4. The best-fit is presented in Fig. 4. The corresponding values of the adjustable parameters are reported in Table 1 . The reproduction of $\delta \Psi$ and $\delta \Delta$ data was very accurate, even in the IR region, for both molecular moieties and both angles of incidence.

Considering the fit uncertainty, the mentioned index $v s$. thickness correlation, and the dispersion of experimental data, we have estimated an overall uncertainty of $\pm 0.2 \mathrm{~nm}$ for $d_{\mathrm{TF}}$, for both dry SAMs.

The $\mathrm{TF}|\mathrm{I}| \mathrm{S}$ model assumption of a uniform film could appear oversimplifying in view of the conceivable different morphologies of the lower and upper regions of the T-OEG film. ${ }^{24}$ However, attempts to refine the model by introducing a grading of $n$ collided with severe problems of mathematical correlation between parameters, without leading to a significant reduction of MSE. Nevertheless, the approach proved useful in the interpretation of in-liquid (TE buffer) SE measurements, which are presented in Fig. 5.

In these measurements the medium/film optical contrast drastically decreased, leading to values of $\delta \Psi$ and $\delta \Delta$ definitely
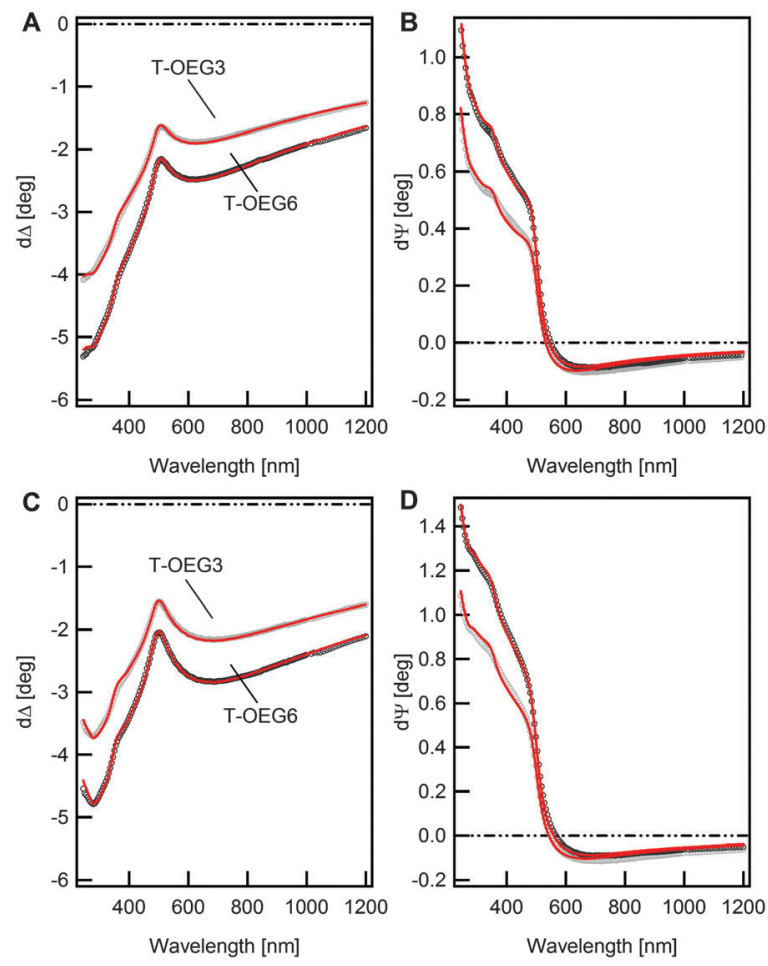

Fig. 4 Comparison between SE data (symbols) for T-OEG3 and T-OEG6 films, and best-fit simulations (lines) at $65^{\circ}$ ( $A$ and $B$ ) and $70^{\circ}(C$ and $D$ ) angles of incidence. Simulations have been calculated according to the TFIIIS model. The fitting procedure was performed on averaged data, the two species of SAMs being considered independently. The corresponding best-fit values of the adjustable parameters are reported in Table 1. 
Table 1 Best fit value and fit uncertainty for the adjustable parameters of the TFIIIS model (for details, see the text); the MSE value for each best fit is also shown. The fitting procedure was performed on averaged data and the two species of SAMs were considered independently

\begin{tabular}{lcc}
\hline & T-OEG3 & T-OEG6 \\
\hline$n_{\mathrm{TF}}$ & $1.36 \pm 0.01$ & $1.38 \pm 0.01$ \\
$B\left(\mu \mathrm{m}^{2}\right)$ & $0.006 \pm 0.001$ & $0.005 \pm 0.001$ \\
$d_{\mathrm{TF}}(\mathrm{nm})$ & $1.9 \pm 0.1$ & $2.6 \pm 0.05$ \\
$d_{\mathrm{I}}(\mathrm{nm})$ & $0.28 \pm 0.01$ & $0.26 \pm 0.01$ \\
$f_{\mathrm{SAM}}(\%)$ & $61 \pm 13$ & $61 \pm 9$ \\
$\operatorname{MSE}$ & 2.2 & 2.1
\end{tabular}

smaller than those found ex situ. Taking into account the increased relative uncertainty, the thickness difference between the two SAMs would appear even questionable. This finding at the first glance appears in striking contrast to both AFM and ex situ SE results. And indeed the simple model used in the case of dry samples is not able to fit the SE data in liquid. In this respect we note that several authors claimed the penetration of water molecules in the film. ${ }^{21,31,32,58}$ Partial hydration of the film could contribute to render the surface of the SAM less defined, thus smoothing the optical contrast between the brush-like part of the film and the ambient. The modelling of complex SAM/water interphases requires an increasing number of parameters to account for e.g. the conceivable grading of water density. The interplay between the mathematical correlation between the fit parameters ${ }^{14}$ and the relatively large, experimental uncertainty makes it hard to achieve a robust, quantitative assessment of the degree of water penetration on the refractive index of the SAM part and, consequently, a robust determination of the thickness. However, an appealing interpretation can be advanced just inspecting the data. Indeed, we find here a situation typical for Immersion Ellipsometry experiments (SIE), which were exploited on semiconductor interfaces. ${ }^{59,60}$ The index matching between the ambient and the outer part of the film tends to optically remove the latter, leading to an enhancement of the sensitivity to the inner more packed part of the film and to the film/substrate interface as well.
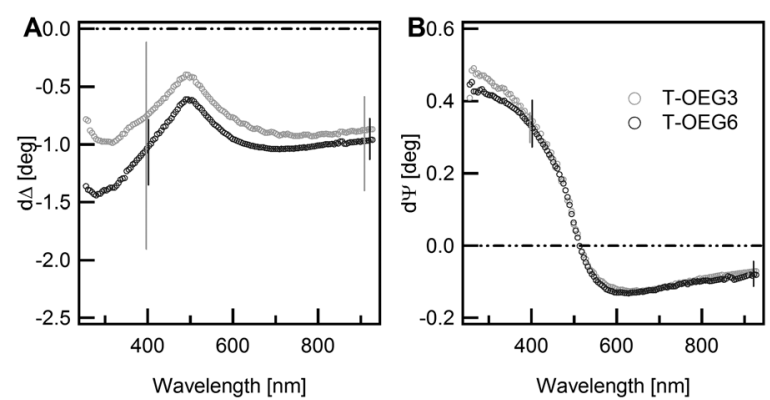

Fig. 5 Ellipsometry difference spectra $\left(\delta \Psi=\Psi_{\text {film }+\mathrm{Au}}-\Psi_{\mathrm{Au}}, \delta \Delta=\Delta_{\mathrm{film}+\mathrm{Au}}-\right.$ $\Delta_{\mathrm{Au}}$ ) of T-OEG SAMS in TE buffer. Measurements were obtained at $65^{\circ}$ angle of incidence and are shown in the range of high transparency of the medium. Thin vertical bars show representative uncertainty in different spectral regions. SAM-induced $\delta \Psi$ and $\delta \Delta$ data are definitely smaller (about a factor of two) than for ex situ measurements. This is mainly due to the limited contrast between the refractive index of the medium (water) and the refractive index of the SAMs, especially the OEG part.
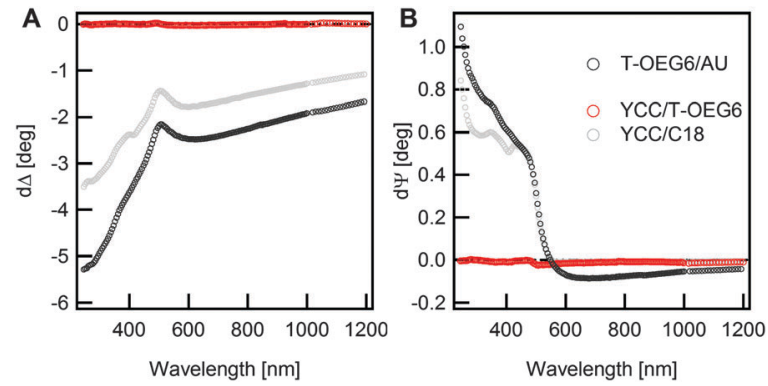

Fig. 6 Ex situ measurements. Dark grey circles: ellipsometry difference spectra $\left(\delta \Psi=\Psi_{\mathrm{TOEG} 6+\mathrm{Au}}-\Psi_{\mathrm{Au}} \delta \Delta=\Delta_{\mathrm{TOEG} 6+\mathrm{Au}}-\Delta_{\mathrm{Au}}\right)$ obtained for TOEG SAMs $\left(65^{\circ}\right.$ angle of incidence; same as Fig. 2). Red curve: ellipsometry difference spectra $\left(\delta \Psi=\Psi_{\mathrm{YCC}+\mathrm{TOEG} / \mathrm{Au}}-\Psi_{\mathrm{TOEG} 6 / \mathrm{Au}}, \delta \Delta=\Delta_{\mathrm{YCC}+\mathrm{TOEG} / \mathrm{Au}}-\right.$ $\left.\Delta_{\text {TOEG6/Au }}\right)$ obtained after exposure of the SAMs to YCC $\left(65^{\circ}\right.$ angle of incidence). Difference spectra are practically null for YCC. Adsorption of YCC onto T-OEG therefore appears absolutely negligible. Difference spectra related to the adsorption of YCC on a C18 SAM are also shown (light grey circles) for comparison.

The similarity between T-OEG3 and T-OEG6 results would then reflect a similar organization and density of the lowest-lying part of the film, especially the C11 part, common to the two molecules. Note that the negative $\delta \Psi$ values above $550 \mathrm{~nm}$ are well visible, quantitatively similar to those determined ex situ; thus the in-liquid measurements provide an elegant confirmation of the existence of a transition layer optically distinct from the film and the substrate.

Finally we present ellipsometry measurements aimed to check the resistance of T-OEG SAMs to unspecific adsorption. Representative data, for T-OEG6, are shown in Fig. 6; results obtained for T-OEG3 SAMs were equivalent. There are no significant differences between SE spectra taken after and before exposure to YCC $\left(\delta \Psi=\Psi_{\mathrm{YCC}+\mathrm{TOEG} 6 / \mathrm{Au}}-\Psi_{\mathrm{TOEG} / \mathrm{Au}}, \delta \Delta=\right.$ $\left.\Delta_{\mathrm{YCC}+\mathrm{TOEG6} / \mathrm{Au}}-\Delta_{\mathrm{TOEG} / \mathrm{Au}}\right)$. Instead, in the case of octadecanethiol (C18) SAM well-defined difference spectra are visible with evident features at about $400 \mathrm{~nm}$ indicating the Soret band of YCC. ${ }^{39}$ Note that for the YCC/C18 case $\delta \Psi$ values in the NIR are substantially null, as expected for the sharp interface related to unspecific adsorption. These findings can also be compared with previous studies reporting on the adsorption of YCC onto bare $\mathrm{Au}^{39}$ and silicon oxide substrates, ${ }^{40}$ where the YCC-induced signal was definitely significant and neat fingerprints of the optical absorption associated with the heme group were detected.

\section{Concluding remarks}

Let us resume the information obtained by just inspecting the difference SE spectra or resorting to the comparison with simulations built on simple models. The measurements confirmed the absence of any intrinsic optical absorption of the T-OEG molecules in the probed wavelength range. Well-defined spectroscopic features in the NIR spectral region, shared by all the thiol/gold systems investigated in our previous studies, specifically provided information on the formation of the $\mathrm{S}-\mathrm{Au}$ interface. The measurements in liquid showed a limited 
sensitivity to the OEG part of the film, indicating a decrease of the refractive index going from the more packed C11 part to the part consisting of flexible OEG chains, eventually penetrated by water. The broadband data provided a sharp picture of the high resistance of T-OEG SAMs to unspecific adsorption of proteins. This result firmly confirms the conclusions of previous studies based on single-wavelength optical methods like SPR, or electrochemical methods. ${ }^{1,23,61-63}$

Regarding the quantitative determination of film thickness the comparison of SE and AFM nanolithography, exploited here for the first time, revealed complementary points of strength. AFM nanolithography displayed the highest potential for in-liquid measurements. Conversely, in-liquid SE measurements proved problematic for a precise determination of the thickness; nevertheless they provided interesting clues about the vertical morphology of the films, which is not accessible to the AFM probe, and is difficult to obtain with other more perturbative methods. Furthermore, the in-liquid SE measurements indicate a refractive index matching between the aqueous medium and the outer part of the SAM, consistent with a disordered configuration of the OEG and/ or the penetration of water amid the OEG strands, factors which are expected to determine the bio-inertness of the film. Indeed a complex water/OEG interphase is a key factor to build models with the ability to describe the non-fouling properties of SAMs. ${ }^{20,27,28,30,32,58,64-66}$ Several studies explicitly suggested the trapping of water molecules into the SAM, eventually correlated to the packing density, as a necessary prerequisite for the resistance properties of OEG. $^{23,29}$

A quantitative assessment of thickness by SE was instead possible on dry samples, thanks to the increased ambient/film optical contrast. This could support previous claims ${ }^{24}$ on the fact that after removal from the fluid the water molecules would remain absorbed mainly at the terminal groups of the SAM. The thickness difference between T-OEG6 and T-OEG3 SAMs derived from the $\mathrm{T}|\mathrm{I}| \mathrm{S}$ model applied to SE data on dry samples is in nice agreement with AFM height measurements. Regarding absolute values (Table 1), the values of $1.9 \pm 0.2$ and $2.6 \pm 0.2$ for T-OEG3 and T-OEG6 SAMs, respectively, are slightly higher than the AFM-based determinations and appear even higher taking the interface layer thickness $(\approx 0.3 \mathrm{~nm})$ into account. Note that conceivable factors may lead to underestimating the absolute thickness such as the imperfect thiol depletion of shaved areas as well as a slight compression of the soft layer under the tip pressure. ${ }^{67}$ On the other hand, the refractory character of T-OEG SAMs cannot rule out some adventitious adsorption from the room ambient, which can lead to a slight overestimation of thickness in ex situ SE measurements. Thickness values are fully compatible with other determinations based on ellipsometry and more perturbative methods such as XPS. ${ }^{7,20,54}$ The resulting T-OEG6 film thickness $\left(d_{\mathrm{TF}}+d_{\mathrm{I}} \approx 2.9\right)$ appears slightly smaller than the estimated length of the molecule $(\approx 3-3.3 \mathrm{~nm}),{ }^{68}$ which is consistent with a relative SAM disorder.

The good agreement between AFM and SE regarding the film thickness strengthens the spectroscopic ellipsometry analysis, helping to attenuate the uncertainty related to the thickness/ index correlation. The refractive index of the T-OEG films, in the 1.35-1.40 range, could appear somewhat lower than those commonly assumed for dense alkanethiol or even T-OEG SAMs. $^{7,34,68}$ In this respect it is worth recalling that the $\mathrm{T}|\mathrm{I}| \mathrm{S}$ model provides an average between the indices of more (C11) and less densely packed (OEG) parts of the film.

The results of our work demonstrate the high potential of the method of cross-checking AFM nanolithography and SE in the analysis of ultrathin organic and biologic films, providing a detailed insight into the subtle issues and pitfalls involved in the thickness determination of soft-matter films, especially transparent ones, down to the limit of a monolayer.

\section{Acknowledgements}

The authors thank Prof. Giacinto Scoles and Dr. Marco Lazzarino for discussions. The authors dedicate this paper to Giacinto Scoles on the occasion of his 80th birthday. This work was supported by the Italian Ministry of Education (grant RBAP11ETKA-005) and the University of Genova.

\section{References}

1 K. Prime and G. Whitesides, Science, 1991, 252, 1164-1167.

2 A. Hucknall, S. Rangarajan and A. Chilkoti, Adv. Mater., 2009, 21, 2441-2446.

3 L. S. Jung, C. T. Campbell, T. M. Chinowsky, M. N. Mar and S. S. Yee, Langmuir, 1998, 14, 5636-5648.

4 D. J. Vanderah, R. J. Vierling and M. L. Walker, Langmuir, 2009, 25, 5026-5030.

5 M. Poksinski and H. Arwin, Thin Solid Films, 2004, 455-456, 716-721.

6 A. Nabok and A. Tsargorodskaya, Thin Solid Films, 2008, 516, 8993-9001.

7 C. Pale-Grosdemange, E. S. Simon, K. L. Prime and G. M. Whitesides, J. Am. Chem. Soc., 1991, 113, 12-20.

8 A. Ulman, Chem. Rev., 1996, 96, 1533-1554.

9 J. C. Love, L. A. Estroff, J. K. Kriebel, R. G. Nuzzo and G. M. Whitesides, Chem. Rev., 2005, 105, 1103-1170.

10 R. M. A. Azzam and N. M. Bashara, Ellipsometry and Polarized Light, North-Holland, New York, 3rd edn, 1977.

$11 \mathrm{H}$. Fujiwara, Spectroscopic Ellipsometry: Principles and Applications, Wiley, Chichester, UK, 1st edn, 2007.

12 F. Bordi, M. Prato, O. Cavalleri, C. Cametti, M. Canepa and A. Gliozzi, J. Phys. Chem. B, 2004, 108, 20263-20272.

13 L. Pasquali, S. Mukherjee, F. Terzi, A. Giglia, N. Mahne, K. Koshmak, V. Esaulov, C. Toccafondi, M. Canepa and S. Nannarone, Phys. Rev. B: Condens. Matter Mater. Phys., 2014, 89, 045401.

14 K. B. Rodenhausen, T. Kasputis, A. K. Pannier, J. Y. Gerasimov, R. Y. Lai, M. Solinsky, T. E. Tiwald, H. Wang, A. Sarkar, T. Hofmann, N. Ianno and M. Schubert, Rev. Sci. Instrum., 2011, 82, 103111.

15 N. A. Geisse, Mater. Today, 2009, 12, 40-45.

16 I. Kopf, C. Grunwald, E. Bründermann, L. Casalis, G. Scoles and M. Havenith, J. Phys. Chem. C, 2010, 114, 1306-1311. 
17 F. Lu, M. Jin and M. A. Belkin, Nat. Photonics, 2014, 8, 307-312.

18 G.-Y. Liu, S. Xu and Y. Qian, Acc. Chem. Res., 2000, 33, 457-466.

19 I. Banerjee, R. C. Pangule and R. S. Kane, Adv. Mater., 2011, 23, 690-718.

20 P. Harder, M. Grunze, R. Dahint, G. M. Whitesides and P. E. Laibinis, J. Phys. Chem. B, 1998, 102, 426-436.

21 A. J. Pertsin and M. Grunze, Langmuir, 2000, 16, 8829-8841.

22 M. Zwahlen, S. Herrwerth, W. Eck, M. Grunze and G. Hähner, Langmuir, 2003, 19, 9305-9310.

23 L. Li, S. Chen, J. Zheng, B. D. Ratner and S. Jiang, J. Phys. Chem. B, 2005, 109, 2934-2941.

24 R. Y. Wang, M. Himmelhaus, J. Fick, S. Herrwerth, W. Eck and M. Grunze, J. Chem. Phys., 2005, 122, 164702.

25 N. Inada, H. Asakawa, Y. Matsumoto and T. Fukuma, Nanotechnology, 2014, 25, 305602.

26 P. S. Johnson, M. Goel, N. L. Abbott and F. J. Himpsel, Langmuir, 2014, 30, 10263-10269.

27 S. Jeon, J. Lee, J. Andrade and P. D. Gennes, J. Colloid Interface Sci., 1991, 142, 149-158.

28 A. J. Pertsin, T. Hayashi and M. Grunze, J. Phys. Chem. B, 2002, 106, 12274-12281.

29 S. Herrwerth, W. Eck, S. Reinhardt and M. Grunze, J. Am. Chem. Soc., 2003, 125, 9359-9366.

30 H. I. Kim, J. G. Kushmerick, J. E. Houston and B. C. Bunker, Langmuir, 2003, 19, 9271-9275.

31 J. Zheng, L. Li, S. Chen and S. Jiang, Langmuir, 2004, 20, 8931-8938.

32 T. Hayashi, Y. Tanaka, Y. Koide, M. Tanaka and M. Hara, Phys. Chem. Chem. Phys., 2012, 14, 10196-10206.

33 L. K. Ista and G. P. Lopez, Langmuir, 2012, 28, 12844-12850.

34 M. Prato, R. Moroni, F. Bisio, R. Rolandi, L. Mattera, O. Cavalleri and M. Canepa, J. Phys. Chem. C, 2008, 112, 3899-3906.

35 M. Canepa, in A Surface Scientist's View of Spectroscopic Ellipsometry, ed. G. Bracco and B. Holst, Springer Series in Surface Sciences, Berlin, Germany, 1st edn, 2013, vol. 51, issue 1, pp. 99-135.

36 H. Hamoudi, M. Prato, C. Dablemont, O. Cavalleri, M. Canepa and V. A. Esaulov, Langmuir, 2010, 26, 7242-7247.

37 M. Canepa, G. Maidecchi, C. Toccafondi, O. Cavalleri, M. Prato, V. Chaudhari and V. A. Esaulov, Phys. Chem. Chem. Phys., 2013, 15, 11559-11565.

38 M. Prato, M. Alloisio, S. A. Jadhav, A. Chincarini, T. SvaldoLanero, F. Bisio, O. Cavalleri and M. Canepa, J. Phys. Chem. C, 2009, 113, 20683-20688.

39 C. Toccafondi, M. Prato, G. Maidecchi, A. Penco, F. Bisio, O. Cavalleri and M. Canepa, J. Colloid Interface Sci., 2011, 364, 125-132.

40 C. Toccafondi, O. Cavalleri, F. Bisio and M. Canepa, Thin Solid Films, 2013, 543, 78-82.

41 C. Toccafondi, L. Occhi, O. Cavalleri, A. Penco, R. Castagna, A. Bianco, C. Bertarelli, D. Comoretto and M. Canepa, J. Mater. Chem. C, 2014, 2, 4692-4698.

42 H. Hamoudi, K. Uosaki, K. Ariga and V. A. Esaulov, RSC Adv., 2014, 4, 39657-39666.
43 P. Gupta, K. Loos, A. Korniakov, C. Spagnoli, M. Cowman and A. Ulman, Angew. Chem., Int. Ed., 2004, 43, 520-523.

44 A. Bosco, F. Bano, P. Parisse, L. Casalis, A. De Simone and C. Micheletti, Nanoscale, 2012, 4, 1734-1741.

45 D. E. Aspnes, E. Kinsbron and D. D. Bacon, Phys. Rev. B: Condens. Matter Mater. Phys., 1980, 21, 3290-3299.

46 Handbook of ellipsometry, ed. H. Tompkins and E. Irene, Andrew, Norwich, 1st edn, 2005.

47 M. Losurdo, M. Bergmair, G. Bruno, D. Cattelan, C. Cobet, A. de Martino, K. Fleischer, Z. Dohcevic-Mitrovic, N. Esser, M. Galliet, R. Gajic, D. Hemzal, K. Hingerl, J. Humlicek, R. Ossikovski, Z. V. Popovic and O. Saxl, J. Nanopart. Res., 2009, 11, 1521-1554.

48 T. W. H. Oates, H. Wormeester and H. Arwin, Prog. Surf. Sci., 2011, 86, 328-376.

49 G. E. Jellison Jr., Appl. Opt., 1991, 30, 3354-3360.

50 J. N. Hilfiker, N. Singh, T. Tiwald, D. Convey, S. M. Smith, J. H. Baker and H. G. Tompkins, Thin Solid Films, 2008, 516, 7979-7989.

51 M. Prato, A. Gussoni, M. Panizza, O. Cavalleri, L. Mattera and M. Canepa, Phys. Status Solidi C, 2008, 5, 1304-1307.

52 K. A. Bell, L. Mantese, U. Rossow and D. E. Aspnes, J. Vac. Sci. Technol., B: Microelectron. Nanometer Struct.-Process., Meas., Phenom., 1997, 15, 1205-1211.

53 J. Shi, B. Hong, A. Parikh, R. Collins and D. Allara, Chem. Phys. Lett., 1995, 246, 90-94.

54 B. Zhu, T. Eurell, R. Gunawan and D. Leckband, J. Biomed. Mater. Res., 2001, 56, 406-416.

55 O. Neuman and R. Naaman, J. Phys. Chem. B, 2006, 110, 5163-5165.

56 J. Märtensson and H. Arwin, Langmuir, 1995, 11, 963-968.

57 G. Gonella, O. Cavalleri, I. Emilianov, L. Mattera, M. Canepa and R. Rolandi, Mater. Sci. Eng., C, 2002, 22, 359-366.

58 M. Tanaka, T. Hayashi and S. Morita, Polym. J., 2013, 45, 701-710.

59 F. Lukeš, W. Knausenberger and K. Vedam, Surf. Sci., 1969, 16, 112-125.

60 Q. Liu, J. F. Wall and E. A. Irene, J. Vac. Sci. Technol., A, 1994, 12, 2625-2629.

61 L. Li, S. Chen and S. Jiang, J. Biomater. Sci., Polym. Ed., 2007, 18, 1415-1427.

62 H. M. Zareie, C. Boyer, V. Bulmus, E. Nateghi and T. P. Davis, ACS Nano, 2008, 2, 757-765.

63 K. Yoshioka, Y. Sato, M. Tanaka, T. Murakami and O. Niwa, Anal. Sci., 2010, 26, 33-37.

64 D. Schwendel, T. Hayashi, R. Dahint, A. Pertsin, M. Grunze, R. Steitz and F. Schreiber, Langmuir, 2003, 19, 2284-2293.

65 M. Östblom, R. Valiokas, P. Konradsson, S. C. T. Svensson, B. Liedberg, M. Garrett and D. L. Allara, J. Phys. Chem. B, 2006, 110, 1830-1836.

66 T. Hirata, H. Matsuno, D. Kawaguchi, T. Hirai, N. L. Yamada, M. Tanaka and K. Tanaka, Langmuir, 2015, 31, 3661-3667.

67 F. Moreno-Herrero, J. Colchero and A. Bar, Ultramicroscopy, 2003, 96, 167-174.

68 R. Valiokas, S. Svedhem, M. Östblom, S. C. T. Svensson and B. Liedberg, J. Phys. Chem. B, 2001, 105, 5459-5469. 